\title{
PROPUESTAS PARA AMPLIAR LA COBERTURA DE SALUD MENTAL INFANTIL EN CHILE
}

\author{
Alejandra Zúñiga-Fajuri², Mónica Zúñiga F. ${ }^{3}$
}

Resumen: Las experiencias tempranas de vida impactan de manera significativa en la arquitectura del cerebro del infante, pudiendo fortalecerla o debilitarla permanentemente. La sintomatología psicológica presente en la infancia no es transitoria, por lo que la intervención temprana supone beneficios directos, tanto sociales como económicos. El presente trabajo propone fundamentar, con base en los hallazgos de la literatura más reciente, la incorporación en Chile de nuevas Garantías Explícitas en Salud, destinadas específicamente a tratar patologías mentales de la infancia. Se argumenta que se trata de una política pública altamente eficiente, capaz de aportar significativamente en la disminución tanto de los costes asociados a Salud Mental como de las brechas de equidad en salud.

Palabras clave: salud mental, infancia, economía de la salud, trauma, depresión

\section{Proposals to expand the coverage of Child Mental Health in Chile}

\begin{abstract}
Early life experiences can make an important impact in the architecture of the infant's brain, which can strengthen or weaken it permanently. The childhood psychological symptomatology is not fleeting, so early intervention involves direct social and economic benefits. This paper offers, based on the most recent findings, the addition of new Health Care Guarantees specifically aimed to treating mental pathologies in childhood. This public policy has been shown to be highly efficient and capable to make a real contribution to the reduction of both, the costs associated with Mental Health and the equity gaps in child health care.
\end{abstract}

Key words: mental health, childhood, health economics, trauma, depression

\section{Propostas para expandir a cobertura de saúde mental infantil no Chile}

Resumo: As experiências iniciais da vida afetam significativamente a arquitetura do cérebro da criança, que pode fortalecê-lo ou enfraquecê-lo permanentemente. A sintomatologia psicológica presente na infância não é transitória, portanto a intervenção precoce envolve benefícios sociais e econômicos diretos. O presente trabalho propóe basear, com base nos achados da literatura mais recente, a incorporação no Chile de novas Garantias Explícitas de Saúde especificamente voltadas ao tratamento de patologias mentais da infância. Argumenta-se que se trata de uma política pública altamente eficiente, capaz de contribuir significativamente para a redução tanto dos custos associados à Saúde Mental quanto das disparidades de equidade em saúde.

Palavras-chave: saúde mental, infância, economia da saúde, trauma, depressão

\footnotetext{
${ }^{1}$ Articulo asociado al Proyecto de Investigación FONDECYT N 1180096. "La Salud Mental Infantil como una nueva Garantía Explícita en Salud".

${ }^{2}$ Universidad de Valparaíso, Chile. Centro de Investigación de Filosofía del Derecho y Derecho Penal, Universidad de Valparaíso, CIFDE-UV, Chile Correspondencia: alejandra.zuniga@uv.cl

${ }^{3}$ Centro de Investigación de Filosofía del Derecho y Derecho Penal, Universidad de Valparaíso, CIFDE-UV, Chile
} 


\section{Introducción}

Se revisa la situación de la salud mental infantil en Chile sobre la base de que, más allá de las actuales iniciativas e intervenciones ejecutadas para mejorar el desarrollo infantil, la Organización Mundial de la Salud (OMS) y el Comité de los Derechos del Niño de las Naciones Unidas han señalado que el cumplimiento de la Convención de las Naciones Unidas sobre los Derechos del Niño está aún pendiente(1). Esta situación requiere no solo insistir en el perfeccionamiento de los actuales planes de salud mental, sino que incorporar al mínimo sanitario, conformado por las llamadas GES (Garantías explícitas en Salud), las principales patologías que afectan a los niños en Chile, garantizando así la disponibilidad de fondos suficientes para su salud mental.

La infancia es un periodo crítico para el desarrollo de la conducta, la capacidad de aprendizaje y la resiliencia del individuo. Numerosos estudios muestran que la sintomatología psicológica presente en la infancia no es transitoria y que, por el contrario, la intervención temprana supone beneficios directos, al abordar los síntomas antes de que se transformen en una conducta crónica maladaptativa(2). Se requieren políticas públicas que den prioridad a la salud mental de los niños, con el fin de evitar que las experiencias tempranas de vida impacten de manera significativa en la arquitectura del cerebro del infante (que pueden fortalecerla o debilitarla permanentemente).

En esta línea, revisaremos la más reciente literatura que ha confirmado los efectos negativos permanentes generados por experiencias de violencia, abusos sexuales, pobreza y abandono, para luego exponer argumentos, tanto económicos como sociales, que fundamentan la incorporación de nuevas Garantías Explícitas en Salud destinadas específicamente a tratar patologías mentales infantiles. Son medidas destinadas a mitigar los daños que las experiencias adversas causan tanto a la infancia y la adultez, como al sistema sanitario en su conjunto.

Finalmente se presenta, con base en la más reciente literatura científica, una propuesta de inclusión de cuatro nuevas patologías en el listado de GES. Se trata de problemas de Salud Mental que, por sus estadísticas, cronicidad e impacto, hacen impres- cindible su incorporación en el mínimo sanitario destinado a salvaguardar el derecho de niñas y niños de Chile.

\section{¿Por qué Salud Mental en la infancia?}

\section{Las porfiadas cifras}

Chile ha realizado un progresivo desarrollo de planes nacionales de salud mental desde el año 2000, aumentando y capacitando a profesionales de salud mental e implementando programas específicos destinados a la infancia, como el programa "Chile Crece Contigo". Sin embargo, los estudios han detectado que Chile sigue siendo uno de los países con la mayor prevalencia de dificultades de salud mental en niños de 1 a 5 años(3). La OMS ha situado a Chile entre los estados con mayor cargara de morbilidad por enfermedades psiquiátricas en el mundo. Según el último Estudio de Carga de Enfermedad y Carga Atribuible, un $23,2 \%$ de los ańos perdidos por discapacidad o muerte (AVISA) están determinados por condiciones neuropsiquiátricas. Para niñas y niños de entre 1 y 9 años un 30,3\% de los AVISA se debe a estas condiciones, proporción que se incrementa a un 38,3\% entre los de 10 a 19 años(4). Sin embargo, el gasto en salud en Chile sigue siendo muy inferior al de la mayoría de los países de la Organización para la Cooperación y el Desarrollo Económicos (OCDE): "Lo que implica que menos del 50\% del gasto total en salud es financiado con medios públicos y una gran parte es pagada directamente por las familias"(5).

Desde la neurología se ha demostrado que las experiencias de estrés tóxico en la infancia no solo pueden afectar los circuitos de interconexión cerebral que se establecen de manera rápida y continua en los primeros años de vida, sino que estas modificaciones pueden trascender a la manera como el cerebro funciona al alterar su arquitectura neurológica(6). Esto se traduce en sobrerreacciones conductuales en la niñez, dificultades de aprendizaje y mermas en la salud mental y física en la vida adulta(7). La mitad de los adultos con patologías psiquiátrica evidencia haber presentado problemas diagnosticados en la infancia y ausencia o insuficiencia de tratamiento terapéutico(8).

La prevalencia psiquiátrica en niñas, niños y 
adolescentes (NNA) es de cuatro de cada diez —38\%—, siendo mayor la prevalencia en niños/ as - 4 a 11 años, 43\% — que en adolescentes -12 a 18 años, 33\%. Además, para casos en que el problema psiquiátrico implicó alguna discapacidad la cifra alcanzó a un $22 \%$. Es decir, uno de cada cinco NNA en Chile presenta algún problema de salud mental acompañado de discapacidad (9), y las ya preocupantes cifras de suicidio se han incrementado(10). Si consideramos, además, que la violencia intrafamiliar es una de las principales fuentes de trastorno mental y que, según UNICEF, un $71 \%$ de los NNA recibe algún tipo de violencia de parte de su padre/madre, y que un $51,5 \%$ es víctima de algún tipo de violencia físi$\mathrm{ca}(11)$, la urgencia de incorporar nuevas GES para la infancia parece innegable.

2. Economía de la Salud: la salud mental infantil es una política pública altamente eficiente

Heckman sostiene que cualquier inversión de capital que no esté dirigida a la primera infancia y jóvenes, puede ser entendida como desvío de recursos desde el punto de vista de la eficiencia(12). La Economía de la Salud ha evidenciado cómo la intervención temprana en salud mental permite atacar los síntomas antes de que se transformen en una conducta crónica. Los últimos estudios publicados en Lancet afirman que la inversión en el tratamiento de la depresión y la ansiedad tiene un rendimiento del 400\%(13). El reciente "Reporte de Políticas Públicas" para la primera infancia de la Universidad de Harvard (que considera 40 años de robusta información científica) constató que las intervenciones de salud mental en niñas y niños expuestos a situación de vulnerabilidad ha sido uno de los factores claves para la disminución del gasto en salud y el éxito de los programas dirigidos a la infancia(14).

En Chile, las enfermedades mentales ocupan, desde 2008, el primer lugar entre las causas de licencias médicas(15). Los costos sociales de las experiencias adversas dependen directamente de la intervención temprana en salud mental, la que funciona como un factor protector que puede reducir de manera importante la huella marcada por el estrés crónico, promoviendo la competencia y adaptabilidad positiva a lo largo de la vida(16).
Esto explica el significativo crecimiento de los programas de intervención en salud mental infantil en los pasados 20 años en los países desarrollados, en los que se ha comprobado que su abordaje de manera preventiva reduce considerablemente los problemas de salud mental en la adultez y sus costos asociados(17). Hay mucha evidencia de que las inversiones dirigidas a promover, prevenir, detectar y tratar tempranamente las enfermedades mentales son rentables y eficaces, produciendo retornos de la inversión inicial a mediano plazo y el autofinanciamiento y disminución de los costos de salud y otras áreas (por ejemplo, la criminalidad), en un plazo menor a 5 años(18). Así, desde el punto de vista económico, la concentración de gastos en la infancia tiene un mejor retorno de la inversión en comparación con el gasto en adultos(12). La estrategia más eficiente de desarrollo del capital humano es el enfoque preventivo en términos emocionales, cognitivos y sociales, en la medida que involucra menos costos que aquellos asociados a remediar e intervenir en los problemas cronificados a través del tiempo.

\section{Propuesta para prestaciones mínimas en salud mental infantil}

Toda política pública de salud requiere sustentarse en la mejor evidencia científica posible, para que los legisladores, en el marco del proceso de creación de normas, puedan contar con toda la información necesaria para, primero, justificar la elección de una determinada política sanitaria y, segundo, proponer los mejores mecanismos para su correcta ejecución en los distintos niveles(19). Puesto que existe controversia respecto del diagnóstico de psicopatologías en la infancia (ya que niñas y niños están en permanente desarrollo), consideramos que el abordaje terapéutico más apropiado en los primeros ańos de vida es el propuesto por la psicopatología del desarrollo, que identifica los factores protectores y de riesgo a los cuales los niños se ven expuestos, para luego diferenciar las conductas problemáticas - que pueden ser solo atrasos del desarrollo- de las que representan propiamente desórdenes de la personalidad(20).

A continuación proponemos la incorporación de cuatro nuevas patologías en el Plan AUGE/GES, considerando la frecuencia, cronicidad y significativas consecuencias en las distintas áreas de fun- 
cionamiento familiar, académico y social de los niños.

i) El trauma (TEPT):

En los pasados 20 años se ha acumulado enorme evidencia sobre cómo las niñas y niños testigos de violencia, o que experimentan violencia a nivel interpersonal, tienen reveladoras dificultades para alcanzar las pautas del desarrollo o maduración esperadas para su edad, quedando más propensos a sufrir trastornos conductuales, estrés postraumático, ansiedad y depresión(21). Las experiencias de violencia y maltrato alteran de manera adversa el desarrollo y funcionamiento neurobiológico, psicológico y social(22), por lo que, desde la psicología y la psiquiatría, se considera que los efectos del trauma y violencia interpersonal en niños y niñas es la causa más común y prevenible de patologías mentales.

En efecto, algunos autores consideran que la psicopatología en la infancia, más que reflejar inmadurez en el desarrollo infantil, es el resultado de la adaptación del individuo a las experiencias traumáticas, desviándose entonces el desarrollo de los parámetros normales. El impacto en el nivel neurológico de las experiencias traumáticas, especialmente en las áreas prefrontal, límbica y amígdala, comprometen negativamente la capacidad de regulación emocional, formación de relaciones interpersonales, tolerancia al estrés y memoria(23).

Una de las sintomatologías fuertemente asociada a la experiencia de eventos traumáticos es la conocida como "trastorno de estrés postraumático" (TEPT). Su característica más relevante son sus síntomas subsecuentes a la experiencia del evento traumático. En este contexto, el "trauma" se entiende como aquella experiencia que expone a la persona a la sensación o amenaza de muerte, acompañada de miedo, pánico y desesperación, $y$, en general, los estudios muestran que niñas y niños expuestos a eventos traumáticos tienen un riesgo similar al de los adultos - es decir, del 20 al $60 \%$ de los casos - de sufrir sintomatología asociada al trastorno de estrés postraumático(24).

La sintomatología más característica del TEPT, como respuesta a la vivencia de un evento traumático, es la de los sentimientos de ansiedad acentua- da, horror intenso y desesperanza. El evento traumático se reexperimenta en el juego, imágenes, pesadillas y pensamientos, apareciendo las conductas evitativas frente a estímulos asociados con el trauma, además de bloqueo de respuestas emocionales positivas, hiperactivación y aislamiento(25) Las niñas y niños que sufren experiencias traumáticas suelen manifestar problemas de conducta y es común que presenten comorbilidad con el trastorno defensivo oposicionista, ansiedad de separación, déficit atencional o depresión(26).

Luego, dada la alta comorbilidad con otros problemas de conducta, es importante tener presente que la aparición de sintomatología psicológica subsecuente a la vivencia de una experiencia traumática en la infancia necesita considerar la presencia del TEPT como principal diagnóstico a ser evaluado.

ii) La depresión:

La experiencia de separación temprana del cuidado materno/paterno, negligencia, deprivación o bien la exposición al cuidado de madres/padres con diagnóstico de depresión, se ha visto asociada a la presencia de depresión infantil desde temprana edad(27). Los síntomas característicos del síndrome depresivo infantil, así como en el adulto, son los asociados al ánimo depresivo y/o irritable, además de desinterés o inhabilidad para disfrutar de las actividades y el juego, alteración del sueño y el apetito(28). Las estadísticas de prevalencia de depresión en niños, niñas y adolescentes en el mundo están alrededor de 6,2\%(29). En Latinoamérica no existen suficientes estudios sobre prevalencia de trastornos psiquiátricos en NNA; sin embargo, algunas estadísticas sitúan los trastornos afectivos en la infancia en Chile en alrededor de un $5,1 \%(9)$.

Los NNA con depresión suelen presentar específicas dificultades en el colegio y en las relaciones familiares y sociales, incluyendo mayor probabilidad de uso y abuso de sustancias. Asimismo, entre más temprana la aparición de depresión, más probable es que el desorden sea crónico y se relacione con problemas de salud mental en la adultez. De este modo, la importancia de la detección temprana y tratamiento está asociada a las significativas consecuencias en múltiples niveles del funciona- 
miento personal, familiar y social, además del aumento del riesgo de muerte por enfermedades o suicidio(30).

iii) La ansiedad (TAG):

Existe una fuerte asociación entre la depresión y la ansiedad, especialmente en el espectro de los trastornos de ansiedad generalizada (TAG)(31). Cuando se han dado trastornos de ansiedad durante la infancia, se genera un riesgo mayor de padecer depresión, abuso de sustancias y otros trastornos psiquiátricos en la adultez. Los TAG afectan al 15-20\% de NNA, representando uno de los más comunes diagnósticos psiquiátricos infantiles(32). En Chile, algunos estudios sobre prevalencia permiten establecer que los trastornos ansiosos son la segunda causa más frecuente de diagnóstico, por debajo solo de los trastornos disruptivos(9). Dentro de los trastornos de ansiedad comunes en la infancia se encuentran los trastornos por ansiedad generalizada, la ansiedad social o fobia social, y la ansiedad de separación.

Las niñas y niños con ansiedad generalizada exhiben síntomas tales como preocupación sobre eventos futuros o pasados, falta de autoconfianza en las propias capacidades, dificultad para controlar la ansiedad en varios contextos y actividades, agitación, fatiga, falta de atención, irritabilidad, dificultad para relajarse y trastornos del sueño. En el caso de que estos síntomas estén presentes ante la separación del cuidador, el síndrome será de "ansiedad de separación". Si, en cambio, la preocupación y ansiedad están asociadas a las relaciones sociales, el diagnóstico pertinente es de "ansiedad o fobia social" (25).

El diagnóstico e intervención temprana de los trastornos de ansiedad es una pieza fundamental de la salud mental infantil, ya que está en el origen de problemas de funcionamiento y salud en general, en las relaciones interpersonales, el desenvolvimiento académico y ocupacional, así como en el riesgo de conductas suicidas(32).

iv) Síndrome de déficit atencional con o sin hiperactividad (TDAH):

El síndrome de déficit atencional, con o sin hiperactividad, posee una prevalencia de aproxima- damente el 5\% de los menores de 18 años en el mundo(33). Este síndrome esta agrupado dentro de los trastornos del desarrollo neurológico y se caracteriza por un patrón persistente de déficit en la atención, con o sin hiperactividad e impulsividad, que interfiere y reduce el funcionamiento en el ámbito social y académico/ocupacional, presentándose los primeros síntomas de inatención y/o inactividad antes de los 12 años(26).

Entre las causas del TDAH están tanto factores genéticos como ambientales (prenatales, perinatales y posnatales). Estos últimos comprenden traumatismos craneoencefálicos en la infancia (que pueden tener origen en el maltrato), infecciones del sistema nervioso central, la prematuridad, el consumo de tóxicos como el alcohol o el tabaco en el embarazo, etc. Es por ello que, en el $70 \%$ de los casos, el TDAH aparece junto a otros trastornos psiquiátricos(34). Entre las comorbilidades más frecuentes destacan: trastorno negativista desafiante, trastorno de conducta, trastorno de ansiedad, trastorno del ánimo, tics, trastorno del aprendizaje, entre otros(35).

Considerando la frecuencia, cronicidad y significativas consecuencias en las distintas áreas de funcionamiento familiar, académico y social, el TDAH aparece como un diagnóstico que requiere temprana detención y tratamiento.

\section{Conclusión}

Si reconocemos la importancia de la salud mental infantil como un componente clave para garantizar la protección, bienestar y calidad de vida de niñas y niños, además de ser un factor protector de la salud mental y funcionamiento en la adultez, es que resulta imperativo poder contar con nuevas patologías garantizadas en salud mental infantil. Esta cobertura debe tener los estándares AUGE, es decir, garantías de acceso universal, oportunidad, cobertura financiera y calidad, con el objeto de completar los planes y programas de salud mental vigentes.

Para avanzar por esta línea, hemos intentado definir las patologías mentales que, por sus estadísticas, cronicidad e impacto, justifican ser parte del listado de garantías explícitas en salud del Plan de Acceso Universal. Así, en primer lugar, proponemos la 
incorporación del trastorno de estrés postraumático (TEPT) que, como vimos, desarrolla síntomas depresivos, ansiosos, disruptivos y otros problemas de conducta en los niños. Estos síntomas deben ser entendidos dentro del trastorno por estrés postraumático como diagnóstico principal.

La depresión infantil y la ansiedad (especialmente en el espectro de los trastornos de la ansiedad generalizada, TAG) son otros importantes diagnósticos que debieran tener cobertura y garantía de atención, dada sus significativas consecuencias en la salud mental y funcionamiento a nivel escolar, familiar y social. Los datos muestran que en Chile la depresión presenta una alta comorbilidad con los trastornos de ansiedad, además de ser el segundo diagnóstico psiquiátrico más frecuente entre niñas, niños y adolescentes, por lo que resulta fundamental considerar la detección y el tratamiento temprano de estas patologías como parte del bienestar en salud mental infantil.
Finalmente, dada la prevalencia, cronicidad y significativas consecuencias a nivel del funcionamiento académico y social, además de una comorbilidad del $70 \%$ con otros trastornos psiquiátricos, el déficit atencional con o sin hiperactividad aparece como otra de las piezas claves dentro del abordaje en salud mental infantil desde un enfoque integral, preventivo y amplio, que garantice el bienestar y promoción del desarrollo saludable de la infancia.

La Economía de la Salud ha comprobado que el abordaje preventivo de estas patologías reduce considerablemente los problemas de salud mental en la adultez y sus costos asociados. La evidencia de que las inversiones dirigidas a promover, prevenir, detectar y tratar tempranamente las enfermedades mentales son rentables y eficaces, permite prever, según vimos, un retorno de la inversión inicial a mediano plazo y el autofinanciamiento y disminución de los costos de salud en un plazo menor a cinco años. La estrategia más eficiente y justa de desarrollo del capital humano es el enfoque preventivo en términos emocionales, cognitivos y sociales, en la medida que es menos costosa que el infructuoso intento de remediar problemas que se han cronificado y que, muchas veces, no se pueden reparar. 


\section{Referencias}

1. Zúñiga-Fajuri A, Zúniga Fajuri M. Mental health policies tackling violation of children's human rights in Chile. The Lancet Child \& Adolescent Health 2019; 3(4): 210-211.

2. Zeanah CH, Zeanah P. The scope of infant mental health. En C. H. Zeanah (ed). The handbook of infant mental health, $3^{\text {rd }}$ ed. New York: The Guilford Press; 2009.

3. Bjarnadottir G, Guðmundsson H, Ahmeti A, Verhulst F, Bilenberg N, Gonçalves M, et al. Behavioral/Emotional Problems of Preschoolers: Caregiver/Teacher Reports from 15 Societies. Journal of Emotional and Behavioral Disorders 2012; 6: 1-14.

4. MINSAL. Informe sobre la base del Instrumento de evaluación del sistema de salud mental de la Organización Mundial de la Salud; 2014. Disponible en: who.int/mental_health/who_aims_country_reports/who_aims_report_chile.pdf (Acceso 12 de diciembre de 2019).

5. Fernández Suárez M, Espinoza Soto A. Salud mental e intervenciones para padres de nińos con trastorno del espectro autista: una revisión narrativa y la relevancia de esta temática en Chile. Revista de Psicología 2019; [S.1.], 37(2): $643-682$.

6. Shonkoff J, Garner A. The lifelong effects of early childhood adversity and toxic stress. Pediatrics 2013; 1: 7.

7. Membride H. Mental health: early intervention and prevention in children and young people. British Journal of Nursing 2016; 25(10): 552-557.

8. Honneyman C. Recognizing mental health problems in children and young people. Pediatric Nursery 2007; 19(8): 38-44.

9. MINSAL. Plan Nacional de Salud Mental. Santiago de Chile; 2017. Disponible en: https://www.minsal.cl/wpcontent/uploads/2017/12/PDF-PLAN-NACIONAL-SALUD-MENTAL-2017-A-2025.-7-dic-2017.pdf (Acceso, 6 enero 2019)

10. Barriga L, Villalta M. Análisis de la Conversación: Psicoterapia de adolescentes depresivos con intento de suicidio en contexto hospitalario. Terapia Psicológica 2019; 37(1): 39.

11. UNICEF. $4^{\circ}$ Estudio De Maltrato Infantil En Chile. Análisis Comparativo 1994-2000-2006-2012. Disponible en: http://unicef.cl/web/wp-content/uploads/2015/07/Maltrato-Infantil.pdf (Acceso 12 de diciembre de 2019).

12. Heckman J. Investing in disadvantaged young children is good economics and good public policy. Research and Economics 2007; 54: 3-56.

13. Chisholm, Sweeny, Sheehan, et al. Scaling-up treatment of depression and anxiety: a global return on investment analysis. Lancet Psychiatry 2016; 3: 415-424.

14. Center on the Developing Child at Harvard University. A Science-Based Framework for Early Childhood Policy: Using Evidence to Improve Outcomes in Learning, Behavior, and Health for Vulnerable Children; 2007. Disponible en: http:// www.developingchild.harvard.edu (Acceso 12 de diciembre de 2019).

15. MINSAL. Estadísticas de Licencias Médicas de Origen Común. Santiago de Chile: Superintendencia de Seguridad Social; 2015, Disponible en: http:/www.supersalud.gob.cl/documentacion/666/w3-propertyvalue-3748.html (Acceso 12 de diciembre de 2019).

16. Knitzer J. Early childhood mental health services: A policy and systems development perspective. En: J. Shonkoff \& S. Meisels (eds.), Handbook of early childhood intervention (2 $2^{\text {nd }}$ ed.). New York: Cambridge University Press; 2000.

17. Nagle G. The Economics of Infant Mental Health. En: CH. Zeanah (Ed). The handbook of infant mental health (3 $\left.{ }^{\text {rd }} \mathrm{ed}\right)$. New York: The Guilford Press; 2009.

18. Knapp, McDaid \& Parsonage. Mental health promotion and mental illness prevention: the economic case. London: Department of Health; 2011.

19. Hawkins B, Parkhurst J. The 'good governance' of evidence in health policy. Evidence \& Policy: A Journal of Research, Debate and Practice 2016; 12(4): 575-592.

20. Angol A, Egger HL. Preschool psychopathology: Lessons for the lifespan. Journal of Child Psychology, Psychiatry and allied Disciple 2007; 48: 17-25.

21. Lieberman A, Ghosh Ippen C, Van Horn P. Don't hit my Tommy. A manual for child-parent psychotherapy with young children exposed to violence and other trauma. Washington: Zero to Three; 2015.

22. Schechter DS, Zeanah CH, Myers MM, Brunelli SA, Liebowitz MR, Marshall RD. Psychological dysregulation in violence-exposed Mathers: salivary cortisol of Mathers with very young children pre-and post-separation stress. Bulletin of the Menninger Clinic 2004; 68(4): 319-336.

23. Buczynski R, Siegel D, Van der Kolk B, Ogden P, Porges S, Lanuis R. Treating trauma master series. National Institute for the Clinical Application of Behavioral Medicine; 2018. Disponible en: https:/www.nicabm.com/program/ treating-trauma-master/ 
Propuestas para ampliar la cobertura de salud mental infantil en Chile - Alejandra Zúñiga-Fajuri, Mónica Zúñiga F.

24. Scheeringa MS. Postraumatic Stress Disorder. En: CH. Zeanah (ed). The handbook of infant mental health, $3^{\text {rd }}$ ed. New York: The Guilford Press; 2009.

25. American Psychiatric Association. Diagnostic and Statistical Manual of Mental Disorders. DSM-5. Washington: American Psychiatric Publishing; 2013.

26. Zero to Three. DC: 0-5. Diagnostic Classification of mental health and developmental disorders of infancy and early childhood. Washington: Zero to Three; 2016.

27. Luby, JL. Depression. En: CH. Zeanah (ed.). The handbook of infant mental health, $3^{\text {rd }}$ ed. New York: The Guilford Press; 2009.

28. Scheeringa MS, Zeanah CH. Reconsideration of harm's way: Onsets and comorbidity patterns in preschool children and their caregivers following Hurricane Katrina. Journal of Clinical Child and Adolescents Psychology 2008; 37: 508-518.

29. Erskine H, Baxter A, Patton G, Moffitt T, Patel V, Whiteford H, Scott J. The global coverage of prevalence data for mental disorders in children and adolescents. Epidemiology and Psychiatric Sciences 2017; 26(4): 395-402.

30. Larraín A, Lobos F. Caracterización del suicidio en Chile: ¿qué nos dicen nuestras estadísticas? En: G. Guajardo (ed.). Suicidios contemporáneos: vínculos, desigualdades y transformaciones socioculturales. Santiago de Chile: Flacso; 2017.

31. Avenevoli S, Knight E, Kessler R, Merikangas K. Epidemiology of depression in children and adolescents. En: J. Abela \& B. Hankin (eds.). Handbook of depression in children and adolescents. New York: The Guilford Press; 2008.

32. Wehry A, Beesdo-Baum K, Hennelly M, Sucheta C, Strawn J. Assessment and treatment of anxiety disorders in children and adolescents. Curr Psychiatry Rep 2015; 17(7): 1-19.

33. Moriyama TS, Cho AJM, Verin RE, Fuentes J, Polanczyk GW. Attention deficit hyperactivity disorder. In: JM Rey \& FD Zepf (eds.). IACAPAP Textbook of Child and Adolescent Mental Health. Geneva: International Association for Child and Adolescent Psychiatry and Allied Professions; 2012: 1-23.

34. Soutullo C. Guía esencial de psicofarmacología del niño y del adolescente. Madrid: Editorial Médica Panamericana; 2011.

35. Steinhausen HC, Novik TS, Baldurson G, Curatola P, Lorenzo MJ, Rodrigues R, et al. Co-existing psychiatric problems in ADHD in the ADORE cohort. Eur Child Adolesc Psychiatry 2006; 15(1): 125-129.

Recibido: 6 de enero de 2020

Aceptado: 13 de enero de 2020 